\title{
PRIMARY BILIARY CHOLANGITIS: PREDICTORS OF POOR RESPONSE TO URSODESOXYCHOLIC ACID AFTER 1 YEAR OF TREATMENT IN MOROCCAN PATIENTS.
}

\author{
Hakima Abid, Inssaf Akoch, Maria Lahlali, Nada Lahmidani, Mounia Elyousfi, Dafr-allah Benajah, \\ Mohamed El Abkari, Adil Ibrahimi \\ Hepato-gastroenterology Unit - University Hospital Hassan II-Fez, \\ Laboratory of human pathology, biomedicine and environment, Faculty of Medicine and Pharmacy of Fez - \\ University Sidi Mohammed Ben Abdellah- Fez- Morocco
}

\section{ABSTRACT}

Introduction: Primary biliary cholangitis (PBC), the new dominance of primary biliary cirrhosis, is a cholestatic disease of autoimmune etiology and represents the leading cause of intra-hepatic cholestasis. Treatment is mainly based on ursodeoxycholic acid. The biological response to treatment is the main predictor of survival without liver transplantation. The Globe-score has been recently validated as the main prognostic factor. Materials and methods: This is a retrospective study carried out in our department collating all cases of PBC followed in consultation. The aim of our work is to research the predictors of poor response to UDCA. Results: 46 patients were collected. The mean age of the patients was 58.82 years, with a predominance of women $(n=43,93.5 \%) .34 .78 \%$ of patients were in the stage of cirrhosis. Anti-M2 mitochondria antibodies were positive in 44 patients (95.65\%). An overlap syndrome was found in 11 patients $(23.9 \%)$. Treatment was based on UDCA combined with corticosteroid therapy and immunosuppressant for overlap syndrome. A biochemical response at 1 year of treatment according to the Paris II criteria was found in $47.8 \%$. The average value of the globe score was 1.35 . A score greater than 0.30 was objectified in 20 cases $(43.47 \%)$. Nineteen cirrhotic patients $(41.30 \%)$ had a globe score $>0.30$. Factors associated with poor response to therapy were: stage of decompensated cirrhosis, elevated pre-therapy total bilirubin greater than $30 \mathrm{~g} / 1$ and hypoalbunemia less than $35 \mathrm{~g} / 1$. The study of the correlation between Globe score and Paris II showed a strong and significant association with a correlation coefficient estimated at 67\%. The Paris II score was significantly correlated with the response to treatment $(\mathrm{p}=0.001)$. Conclusion: In accordance with the data in the literature, the globe-score and Paris II are two similar predictive means for evaluating the response at 1 year of treatment in Moroccan context.

Keywords: Morocco, Predictors of response, Primary biliary cholangitis, Ursodeoxycholic Acid (UDCA).

\section{Corresponding Author:}

Hakima Abid, MD.

Affiliation: Hepato-gastroenterology Unit, Hassan II $^{\mathrm{d}}$ University Hospital, Fez, Morocco.

E-mail: abidhakima@hotmail.com

Copyright (C) 2012- 2021 H. Abid. This is an open access article published under Creative Commons Attribution Non Commercial- No Derives 4.0 International Public License (CC BY-NC-ND). This license allows others to download the articles and share them with others as long as they credit you, but they can't change them in any way or use them commercially.

*****Published in 2021 .

doi: 10.46327/msrjg.1.000000000000---

doi url: https://doi.org/10.46327/msrjg.1.000000000000----

\section{INTRODUCTION}

Primary biliary cholangitis (PBC) or formerly called "primary biliary cirrhosis" is a worldwide recognized chronic inflammatory cholestatic disease of the liver, with an autoimmune mechanism that progressively destroys the small and medium intra-hepatic bile ducts. The prevalence of $\mathrm{PBC}$ is 10 to 40 per 100,000 
populations. It is a rare disease that mainly affects women between the ages of 40 and 60 , but it can be seen among women of different ages as well as among men [1]. In women over 40, the prevalence is 1 in 1000 [1]. This female predominance remains unexplained. The pathogenesis is not fully understood at this time, but it seems to be the result of the interactions between genetic and environmental factors. The PBC is reflected in the foreground by a biological cholestasis, the presence of anti-mitochondrial M2 antibodies (AMA-M2) leading to the diagnosis.

The goal of treatment is to prevent progressive liver disease and then progression to cirrhosis and its complications, including portal hypertension, gastrointestinal bleeding and hepatocellular carcinoma, and thereby improve the quality of life of patients. Ursodeoxycholic Acid (UDCA) at a dose of 13 to $15 \mathrm{mg} / \mathrm{kg} /$ day is now the gold standard treatment with proven efficacy on clinical, biology, histological lesions, as well as patient survival. The biological response to UDCA is considered to be the main prognostic factor during PBC. The most widely used definition of the biological response is that of Paris II [2]. Forty percent of patients with PBC are non-responders to UDCA, and their survival with transplantation has been shown to be significantly lower than that of responders [2].
The Globe score has been recently identified as a simple, inexpensive blood test that has been validated as a prognostic factor during PBC. According to several studies, it has shown better performance for the prediction of death or liver transplantation in comparison with the criteria of Paris I and II. Compared to the latest criteria, the Globe score improves the overall classification of patients in the low to high risk group by nearly $10 \%$ [3].

The aim of our work is to study the correlation between Globe score and the Paris II criteria and to find the predictors of poor response to UDCA.

\section{MATERIALS AND METHODS}

This is a retrospective analytical and descriptive study involving 46 patients carried out in our Hepatogastroenterology Department of the Hassan $\mathrm{II}^{\mathrm{d}}$ University Hospital in Fez, spanning a period of 8 years [March 2012 until March 2020]. All patients with PBC associated or not with autoimmune hepatitis (AIH) were included. The medical records of these patients were retrospectively reviewed using the currently accepted diagnostic criteria for each disease (Table I).

Table I: PBC and AIH Diagnostic criteria

\begin{tabular}{cc}
\hline Diagnostic criteria for PBC & Diagnostic criteria for AIH \\
\hline Serum PAL level $\geq 2$ times the ULN or GGT $\geq 5$ ULN & Serum level of ALT or AST $\geq 5$ times the ULN \\
\hline The presence of AMA-M2 at a significant rate $\geq 1 / 80$. & $\begin{array}{c}\text { A level of IgG } \geq 2 \text { times the ULN or the presence } \\
\text { of ASMA at a significant level } \geq 1 / 80 .\end{array}$ \\
\hline
\end{tabular}

Destructive lymphocytic cholangitis on the histology. The presence of interface hepatitis on the histology.

AMA-M2: Anti-Mitochondrial M2 Antibodies; ASMA: Anti-smooth muscle antibodies; ALT: alanine transferase test; AST: aspartate aminotransferase test; ULN: upper limit of normal; IgG: immunoglobulin G; PAL: alkaline phosphatase; GGT: gamma-glutamyl transpeptidase.

Patients with other cause of chronic liver disease including alcoholic hepatitis, hepatitis $\mathrm{B}$ and $\mathrm{C}$ were excluded from this study.

The data extracted from the patient files included: Age, gender, medical history, symptoms (pruritus, asthenia, jaundice, abdominal pain, etc.), date of diagnosis, presence of an associated autoimmune disease, biological results and immunological workup, results of morphological examinations including abdominal ultrasound and esogastroduodenal fibroscopy, noninvasive means for assessing fibrosis level, results of liver biopsy, type of treatment, duration of treatment, response to treatment at 1 year and the presence of relapse or complications. Paris II criteria, as well as the GLOBE score were used for the response to treatment. Data entry and analysis were studied with SPSS software. A p value $<0.05$ was considered statistically significant. The analysis of correlations between quantitative variables was done using linear logistic regression. 


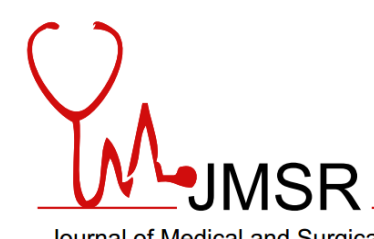

Journal of Medical and Surgical Research

\section{RESULTS}

Forty-six patients were collected during the study period. The mean age of the patients was 58.82 year old [24-83]. Most of the patients were female $(n=43$, 93.5\%). The mean duration of the disease was 6 years. At the time of the first treatment, the clinical symptoms were dominated by pruritus $(\mathrm{n}=26,56.52 \%)$-and asthenia $(n=24,52.17 \%)$. Jaundice was only found in ten patients $(21.73 \%)$. Sixteen patients $(34.78 \%)$ were in the stage of cirrhosis.

The diagnosis of PBC was suspected based on clinical features and the discovery of cholestasis in biology. Cholestasis syndrome was defined by an increase in GGT and PAL in all of our patients. AMA-M2 were positive in 44 patients $(95.65 \%)$.

In patients with overlap syndrome, the immunological workup demonstrated a profile combining AMA- M2 and ANA in 50\% of cases. The clinical and biological characteristics of the disease are summarized in the Table II.

Table II: Clinical and biological characteristics of PBC.

\begin{tabular}{lcc}
\hline Variables & $\mathrm{n}$ & $(\%)$ \\
\hline Middle age & 58,82 year old \\
Female patients & 43 & $93,5 \%$ \\
Average duration of the disease: & 6 years & \\
Antecedents: & 8 & $17,39 \%$ \\
- Familial history of PBC & 28 & $60,86 \%$ \\
- Familial history of autoimmune disease & & \\
Symptoms: & 3 & $6,52 \%$ \\
- Asymptomatic & 26 & $56,52 \%$ \\
- Pruritus & 24 & $52,17 \%$ \\
- Asthenia & 10 & $21.73 \%$ \\
- Jaundice & & \\
Stage of cirrhosis: & 11 & $23.91 \%$ \\
- Compensate & 5 & $10,86 \%$ \\
- Decompensated & 46 & \\
Cholestasis syndrome in biology tests & 9 & $100 \%$ \\
Cytolysis greater than 5 times normal & 12 & $19,56 \%$ \\
Hypoalbunemia less than 35g / 1 & \multicolumn{3}{c}{$26,08 \%$} \\
Immunological assessment: & 26 & \\
- Hypergammaglobinemia & 23 & $56,52 \%$ \\
- AAN positive & 44 & $50 \%$ \\
- AMA-M2 positive & & $95,65 \%$ \\
\hline
\end{tabular}

$\mathrm{n}=$ number of patients; ANA: Antinuclear Antibodies; AMA-M2: Anti-Mitochondrial Antibodies M2.
Associated autoimmune diseases to PBC in our study are showed in Table II.

Table II: Autoimmune diseases associated with PBC in our patients series.

\begin{tabular}{lcc}
\hline Associated autoimmune diseases & $\mathrm{n}$ & $(\%)$ \\
\hline Autoimmune hepatitis & 11 & $23,9 \%$ \\
Diabetes & 7 & 15,21 \\
& & $\%$ \\
Dysthyroidism & 4 & $8,69 \%$ \\
Celiac disease & 2 & $4,34 \%$ \\
Rheumatoid arthritis & 2 & $4,34 \%$ \\
Vitiligo & 1 & $0,21 \%$ \\
Sarcoidosis & 1 & $0,21 \%$ \\
\hline (n= number of patients); & &
\end{tabular}

An overlap syndrome associating $\mathrm{PBC}$ and autoimmune hepatitis $(\mathrm{AIH})$ was found in 11 patients (23.9\%).The histological lesions of overlap syndrome associate in varying degrees the histological signs of $\mathrm{PBC}$ and AIH. In our series, Liver puncture biopsy (LPB) was performed in 9 patients with overlap syndrome and objectified the signs of PBC as well as the signs of hepatocytic necrosis in $35 \%$ of patients. All patients received treatment with UDCA at a dose of 13 to $15 \mathrm{mg} / \mathrm{kg} / \mathrm{day}$. In the event of overlap syndrome, eight patients $(17.39 \%)$ were put on corticosteroid therapy associated with UDCA and three patients $(6.52 \%)$ were put on corticosteroid therapy associated with Azathioprine at a dose of 2 to $2.5 \mathrm{mg} / \mathrm{kg} / \mathrm{day}$. The mean duration of patient followup was 69 months [12-130 months]. A biochemical response at 1 year of treatment according to the Paris II criteria was found in $47.8 \%$. The average value of the globe score was 1.35 . A score greater than 0.30 is objectified in 20 cases (43.47\%). Nineteen cirrhotic patients $(41.30 \%)$ had a globe score> 0.30. In univariate and multivariate analysis, the factors significantly associated to poor response to UDCA at 1 year of treatment in our patients were: The stage of decompensated cirrhosis, poor adherence to treatment, a high level of pre-therapeutic total bilirubin greater than $30 \mathrm{~g} / 1$ and hypoalbunemia less than $35 \mathrm{~g} / 1$ (Table III). 
Table III: Predictors of poor response to UDCA in univariate and multivariate analysis.

\begin{tabular}{lcc}
\hline Variables & Varied uni analysis & Multivariate analysis \\
\hline The stage of decompensated cirrhosis & $\mathrm{p}=0,006$ & $\mathrm{p}=0,001, \mathrm{OR}=5,20[1,424-8,36]$ \\
Advanced age of diagnosis & $\mathrm{p}=0,55$ & \\
A high level of pre-therapeutic total bilirubin & $\mathrm{p}=0,05$ & $\mathrm{p}=0,05, \mathrm{OR}=3,20[1,249-6,412]$ \\
Hypoalbunemia & $\mathrm{p}=0,008$ & $\mathrm{p}=0,001, \mathrm{OR}=7,90[2,456-11,56778]$ \\
Poor adherence to treatment & $\mathrm{p}=0,022$ & $\mathrm{p}=0,005, \mathrm{OR}=8,450[1,789-20,787]$ \\
\hline
\end{tabular}

The study of the correlation between Globe score and Paris II showed a strong and significant association with a correlation coefficient estimated at $67 \%$. The Paris II score was significantly correlated with the response to treatment $(\mathrm{p}<0.001)$.

\section{DISCUSSION}

PBC is a rare disease whose prevalence remains unknown. It is underestimated due to the presence of minimal asymptomatic forms. The disease affects roughly a woman over 40 years / miles [4]. The epidemiology of PBC has been studied extensively in Western countries, as opposed to Africa where epidemiological data are limited. The prevalence in Europe, North America, Asia and Australia is between 19.1 and 402 cases / million population. The incidence is between 0.33 and 5.8 / million habitants / year [5]. In our series, we have noted a female predominance, which matches the data in the literature. Witt-Sullivan et al reported a sex ratio F / M of 13 in Canada [6]. Kapelman et al reported an F / $\mathrm{M}$ sex ratio of 9 in the USA [7].

In the recent years, the diagnosis has increasingly been made at the asymptomatic stage [7]. The diagnosis is suspected when cholestasis is found on a routine laboratory test or during an immunological test requested in the context of another autoimmune disease. Fox et al were the first to describe asymptomatic PBC in 1973 [8].

In symptomatic forms, the most reported functional signs were asthenia, pruritus and jaundice. Seventyfive percent of our patients were symptomatic. Pruritus, asthenia and jaundice were the most frequent signs, found respectively in $56.52 \%, 52.17 \%$ and $21.73 \%$. Springer et al [8] found that the symptoms are identical regardless of the gender except pruritus which was significantly more frequent in women.

The presence of type M2 AMAs is specific for PBC. These autoantibodies appeared several years before the onset of clinical and laboratory signs of PBC. The rate doesn't correlate with gender, histologic stage or disease severity, and has no prognostic value. In our study, AMA-M2 were positive in 44 patients, which is in agreement with the data found in the literature [7, $11,12]$. Various treatments with immunosuppressant and anti-inflammatory drugs (corticosteroids, Azathioprine, D-penicillamine, chlorambucil, methotrexate, colchicine, ciclosporin) have been the focus of several controlled studies. In 1987, UDCA was proposed as a new therapeutic approach based on the hypothesis that liver damage is secondary to the intracellular accumulation of toxic endogenous bile acids. UDCA is now the gold standard for PBC; it improves liver cholestasis, slows histological progression and prolongs patient survival. Corpechot et al [4] have notably demonstrated a survival rate similar to that of the general population in patients at the initial stage of the disease responding to treatment with UDCA. The exact definition of the biological response is a subject of controversy and several criteria are described in the literature. $40 \%$ of patients with PBC are non-responders to UDCA and their survival without liver transplantation has been shown to be significantly lower than that of responders [2, 4]. Most authors agree that UDCA has more beneficial therapeutic effects in the early stages (I and II) of the disease $[13,14]$.

A common cause of resistance to UDCA is the presence of an overlap syndrome associating AIH with PBC. Treatment for the overlap syndrome consists of the combination of UDCA with the immunosuppressive therapy usually recommended for AIH (corticosteroids and Azathioprine). It should be noted that there is no consensus on the exact management of patients with overlap syndrome. The American Association for the Study of Liver Diseases (AASLD) recommends tailoring treatment based on the predominant histological lesion [15]. The European Association for the Study of the Liver (EASL) indicates that immunosuppressive therapy should be initiated in severe interface hepatitis and discussed in moderate interface hepatitis [16].

$\mathrm{PBC}$ is a severe disease progressing to cirrhosis and its complications. Without treatment, the median survival without liver transplantation is estimated at 10 years. After the introduction of UDCA, several scores were proposed, mostly based on the rate of PAL after 
treatment. The two most recent scores based on large multicenter samples are: the GLOBE score and the UK-PBC score (Table IV). They take into account parameters at diagnosis and at 1 year of treatment with UDCA and have proven their superiority in predicting death and liver transplantation compared to other models, including the Paris I criteria [16].

Table IV: Globe score \&UK-PBC score parameters [17, 18].

\begin{tabular}{lll}
\hline Scores & \multicolumn{2}{l}{ Parameters at the start of treatment with UDCA } \\
\hline \multirow{3}{*}{ GLOBE } & & - Total bilirubin \\
& - Age & - Alkaline phosphatases \\
& & - Albumin \\
& & - Platelets \\
\hline \multirow{2}{*}{ UK-PBC } & - Albumin & - Bilirubin \\
& - Platelets & - ASAT or ALAT \\
\hline
\end{tabular}

In our series, the study of the correlation between Globe score and Paris II showed a strong and significant association with a correlation coefficient estimated at $67 \%$. The Paris II score was significantly correlated with response to treatment. N. Elleuch et al. presented similar results showing significant correlation between score and both response to treatment $(\mathrm{p}<0.001)$ and mortality $(\mathrm{p}=0.04)$ [19]. Lammers WJ et al. showed better identification of patients responding insufficiently to UDCA, which is essential to specify the predictive factors of the inadequate response to treatment as highlighted in our study [17].

In our patients, the predictive factors of the poor response to UDCA in univariate and multivariate analysis were: The stage of decompensated cirrhosis, poor adherence to treatment, a high level of pretherapeutic total bilirubin and hypoalbunemia, which is in agreement with literature $[4,5]$. The main factors found in several other studies [4] were: An elevated total bilirubin level at the start of treatment, which is consistent with our series, an advanced histological stage greater than or equal to 3 , the measurement of the hardness of the liver greater than $9.6 \mathrm{KPa}$ and the existence on the LPB of a significant interface hepatitis (segmental and focal in the majority of portal or circumferential spaces in more than $50 \%$ of them) and increased liver hardness $>2.1 \mathrm{kPa} /$ year. N. Elleuch showed that the factors associated with a poor response were: the stage of cirrhosis at diagnosis, an associated autoimmune disease and the pre- Therapeutic elevated total bilirubin [19].

\section{LIMITATIONS}

The presence of overlap syndrome in $23.9 \%$ of our patients' series may be considered as a selection bias. Also, our study is retrospective with limited number of patients. National multicenter study with a significant number of included patients may help to establish the profile of PBC in Moroccan patients with a better evaluation of Paris II and Globe scores in Moroccan context.

\section{CONCLUSION}

The stage of decompensated cirrhosis, poor adherence to treatment, a high level of pre-therapeutic total bilirubin greater than $30 \mathrm{~g} / 1$ and hypoalbunemia less than $35 \mathrm{~g} / 1$ seem to be significant predictive factors of response to UDCA in Moroccan patients. Also, our study showed a good correlation between the globe score and Paris II with better sensitivity and specificity in the Moroccan context. The prognosis of patients with $\mathrm{PBC}$, regardless of disease stage, who have been treated with UDCA for one year can be easily determined using a derived and de novo validated risk calculation. The Globe score provides internationally representative data to quantify the needs of low- and high-risk patients with PBC. The Globe score therefore complements efforts to develop and implement a more layered, evidence-based approach to the management of patients with $\mathrm{PBC}$.

However, other studies will be necessary to deepen this diagnostic score and make it more accessible in current practice.

\section{LIST OF ABBREVIATIONS:}

- $\quad$ AMA-M2 : Anti-Mitochondrial M2 Antibodies

- UDCA : Ursodeoxycholic Acid

- $\quad$ AIH : Autoimmune Hepatitis

- $\quad$ LPB :Liver Puncture Biopsy

- $\quad$ PBC : Primary Biliary Cholangitis

- PAL : Alkaline Phosphatase

- GGT : Gamma-Glutamyl Transpeptidase

\section{CONFLICT OF INTEREST:}

There are no conflict of interest or funding to declare. 


\section{REFERENCES}

1. Boonstra K, Kunst AE, Stadhouders PH, et al. Rising incidence and prevalence of primary biliary cirrhosis: a large population-based study. Liver Int. 2014; 34: e31-8.

2. Lammers WJ, Hirschfield GM, Corpechot C, Nevens F, Lindor KD, Janssen HL, et al. Development and validation of a scoring system to predict outcomes of patients with primary biliary cirrhosis receiving ursodeoxycholic acid therapy. Gastroenterology 2015; 149: 1804-1812.

3. Marco Carbon et al Clinical Application of the GLOBE and United Kingdom-Primary Biliary Cholangitis Risk Scores in a Trial Cohort of Patients With Primary Biliary Cholangitis. Hepatology Communications, 2018 ; II (6)

4. Corpechot C. La cirrhose biliaire primitive. Post'U 2013; 147-154.

5. Invernizzi P, Selmi C, Ranftler C, Podda M, Wesierska-Gadek J. Antinuclear antibodies in primary biliary cirrhosis. Semin Liver Dis. 2005; 25 (3): 298 310.

6. Witt-Sullivan H, Heathcote J, Cauch K, Blendis L, Ghent C, Katz A, et al. The demography of primary biliary cirrhosis in Ontario, Canada. Hepatology. 1990; 12 (1) :98- 105.

7. Kapelman B, Schaffner F. The natural history of primary biliary cirrhosis. Semin Liver Dis. 1981; I(4): 273-81.

8. Prince M, Chetwyn A, Craig W, Metcalf J and James O. Asymptomatic primary biliary cirrhosis: clinical feature, prognosis and symptom progression in a large population based cohort. Gut 2004; 53: 865-70.

9.Fox RA, Scheuer PJ, Scherlock S et al. Asymptomatic PBC. Gut 1973; 14: 444-7.

10. Springer J, Cauch-Dudek K, Orourke K, Wanless IR et Heathcote J. Asymptomatic primary biliary cirrhosis: A study of naturel history and prognosis. Am J Gasteroenterol 1999; 94: 47-53.

11. Marschall H-U, Henriksson I, Lindberg S, Söderdahl $\mathrm{F}$, Thuresson $\mathrm{M}$, Wahlin $\mathrm{S}$, et al. Incidence, prevalence, and outcome of primary biliary cholangitis in a nationwide Swedish population-based cohort. Sci Rep [Internet]. Available from: https://www.ncbi.nlm.nih.gov/pmc/articles/PMC6687 809/

12. N. Maamouri, S. Chouaib, L. Abbes, H. Ouerghi, F. Ben Hriz, N. Belkahia, et al. La cirrhose biliaire primitive en Tunisie: à propos de 43 cas. Gastroentérologie B, hôpital la Rabta, Tunis 2008.
13. Gong Y, Huang Z, Christensen E, Gluud C. Ursodeoxycholic acid for patients with primary biliary cirrhosis: an updated systematic review and metaanalysis of randomized clinical trials using Bayesian approach as sensitivity analyses. Am J Gastroenterol. 2007; 102 (8): 1799-807.

14. Corpechot C, Carrat F, Bahr A, Chrétien Y, Poupon RE, Poupon R. The effect of ursodeoxycholic acid therapy on the natural course of primary biliary cirrhosis. Gastroenterology. 2005;128 (2): 297-303.

15. Lindor KD, Bowlus CL, Boyer J, Levy C, Mayo M. Primary Biliary Cholangitis: 2018 Practice Guidance from the American Association for the Study of Liver Diseases. Hepatology. 2018; hep.30145.

16. Hirschfield GM, Beuers U, Corpechot C, Invernizzi P, Jones D, Marzioni M, et al. EASL Clinical Practice Guidelines: The diagnosis and management of patients with primary biliary cholangitis. Journal of Hepatology. 2017; 67 (1): 145-72.

17. Lammers WJ, Hirschfield GM, Corpechot C, Nevens F, Lindor KD, Janssen HLA, et al. Development and Validation of a Scoring System to Predict Outcomes of Patients With Primary Biliary Cirrhosis Receiving Ursodeoxycholic Acid Therapy. Gastroenterology. 2015; 149 (7): 1804-1812.e4.

18. Carbone M, Sharp SJ, Flack S, Paximadas D, Spiess K, Adgey $\mathrm{C}$, et al. The UK-PBC risk scores: Derivation and validation of a scoring system for long-term prediction of end-stage liver disease in primary biliary cholangitis. Hepatology. 2016;63 (3): 930-50

19. N. Elleuch, B. Hasnaoui, W. Dahman et al. Corrélation entre le score Globe et la réponse à l'acide ursodésoxycholique au cours de la cholangite biliaire primitive. JFHOD 2018 (P001008); https://www.snfge.org/content/correlation-entre-lescore-globe-et-la-reponse-lacide-ursodesoxycholiqueau-cours-de-la 